\title{
Enantioselective metabolism of triadimefon and its chiral metabolite triadimenol in lizards
}

\author{
Qiuxuan Shen, Jitong Li, Peng Xu, Wei Li, Guoqiang Zhuang, Yinghuan Wang* \\ Research Center for Eco-Environmental Sciences, Chinese Academy of Sciences, 18 Shuangqing Road, Haidian District, Beijing 100085, PR China
}

\section{A R T I C L E I N F O}

\section{Keywords:}

Eremias argus

Enantioselectivity

Triadimefon

Triadimenol

\begin{abstract}
A B S T R A C T
Chinese lizards (Eremias argus) were exposed to separated $R$-(-)-triadimefon, $S$ - $(+)$-triadimefon and racemic triadimefon to evaluate enantioselective accumulation of triadimefon. After single oral administration of $R-$ (-)-triadimefon, $S$ - $(+)$-triadimefon and racemic triadimefon, the time-concentration curves in different tissues were found to be different. Triadimefon enantiomers crossed the blood-brain barrier and brain is a main target organ. The residues of triadimefon enantiomers in fat were highest after $24 \mathrm{~h}$ indicating that fat was the main tissue of accumulation. In racemic triadimefon exposure group, the enantiomer fractions of $R$-(-)-triadimefon in different tissues showed that the differences between $R$-(-)-triadimefon and $S-(+)$-triadimefon were significant in absorption and metabolism, but the differences became smaller in exclusion and accumulation. From the results of mathematical models, $S$ - $(+)$-triadimefon was absorbed and eliminated faster than $R$-(-)-triadimefon, and $R$-(-)-triadimefon was easily distributed in the tissues and more easily converted into its metabolites. Furthermore, among the four enantiomers of triadimenol, $S R$-(-)-triadimenol produced by $S$ - $(+)$-triadimefon may have the highest fungicidal activity and the strongest biological toxicity, $R R-(+)$-triadimenol produced by $R$-(-)-triadimefon was most likely to bioaccumulate in lizard. Identifying toxicological effects and dose-response relationship of $S R$-(-)-triadimenol and $R R-(+)$-triadimenol will help fully assess the risk of TF enantiomers use in the future. The results enrich and supplement the knowledge of the environmental fate of triadimefon enantiomers.
\end{abstract}

\section{Introduction}

Approximately $30 \%$ of organic pesticides are chiral compounds and consist of two or more enantiomers (Ulrich et al., 2012). In China, chiral pesticides accounted for all pesticides ratio of $40 \%$ (Ye et al., 2010). The enantioselectivity may occur while the enantiomer binding to structure-sensitive biological receptors (Dong et al., 2013). Due to the action of enzymes, organic substances or other chiral macromolecules, the enantiomers can exhibit significant differences in chiral environments, especially in biological properties (Qi et al., 2016; Zhang et al., 2016). The chiral enantiomers may have identical physic-chemical properties, but the bioactivity processes like uptake, distribution, metabolism, bioaccumulation and toxicity may be completely different (Buser et al., 1992; Zadra et al., 2002; Cai et al., 2008). Now, most of the chiral pesticides are produced, marketed and used as racemates. The proportion of chiral pesticides that can separate the single enantiomeric forms with optical purity is only about 7\% (Zhang et al., 2003). Consideration the probable difference between chiral enantiomers, it is inappropriate to think of them as a single compound in traditional risk assessment (Wong, 2006).

Triadimefon(TF) and triadimenol(TN) are two registered broadspectrum systemic fungicides, triazole-derived molecule extensively used in the field (Crowell et al., 2011). Triadimefon [(RS)-1-(4chlorophenoxy)-3, 3-dimethyl-1- (1H-1, 2, 4-triazol-1-yl) butan-2-one, CAS No.: 43121-43-3] has been commonly used for the control of rusts, powdery mildews, and other fungal pests on fruits, cereals, turf, vegetables, shrubs and trees (Kenneke et al., 2009; Roberts and Hutson, 1999). Triadimenol [(1RS, 2RS; 1RS, 2SR)-1-(4-chlorophenoxy)-3, 3- dimethyl-1-(1H-1, 2, 4-triazol-1-yl) butan-2-ol, CAS No: 55219-65-3], a metabolite of TF by reduction of a carbonyl group to its corresponding alcohol. TN is considered to have a greater fungicidal activity than TF (Liang et al., 2013). TF and TN have already been categorized as "possible human carcinogens" (U.S. Environmental Protection Agency, 2006). Previous studies have shown that there was a potential threat to mammals and aquatic animals due to the wide residue of TF and its metabolites in soil, sediments, natural water and agricultural products (Li et al., 2011, 2014). It has been pointed that excessive TF and TN use could cause teratogenic effects on rat embryos

\footnotetext{
* Corresponding author

E-mail address: yhwang@rcees.ac.cn (Y. Wang).
} 
(Menegola et al., 2000), neurotoxicity in rodents (Moser and Macphail, 1989). Other studies proved that TF is a cytochrome p450 enzyme inhibitor that prevents $17 \beta$-estradiol from the synthesis of testosterone. This suggests that TF may have reproductive toxicity in animals and even humans (Brandelavridsen et al., 2008; Chu et al., 1995; Vinggaard et al., 2000).

The intensification and expansion of agriculture has accelerated the risk of wildlife exposure (Wagner et al., 2015). In China, the abuse of triazole pesticides (Lin et al., 2008) lead to a large amount of TF and TN enter into the cultivated soils. This poses a threat to wildlife living in soil, such as reptiles. Reptiles are vital in maintaining ecological balance, accounting for $28 \%$ of known vertebrate species (Gervais, 2011). Pesticides are considered to be one of the major factors contributing to the global recession of reptiles (Gibbon et al., 2000; Randhawa et al., 2014). Past attention has focused on measuring the body burden of various contaminants on wild reptiles to understand the historical exposure of a given population (Merwe et al., 2009; Moss et al., 2009; Simoniello et al., 2010). But the effects of contaminants on the actual risk and population level of reptiles are still largely unknown (Weir et al., 2010). Lizards are natural enemies of crops and are important but neglected in the agricultural system. Lizards are the most commonly used reptile species in pesticide toxicology, due to their living habits (Buono et al., 2007; Holem et al., 2008). Taking into account the small size, gentle temperament and suitable for using in laboratory, Chinese lizards (Eremias argus) were used in our study. $E$. argus are widely distributed in the north of Yangtze River region, including China's major agricultural areas in North China Plain and Northeast Region. Extensive use of TF and TN in these areas (Lin et al., 2008) has been a huge threat to the E. argus.

$\mathrm{TF}$ and TN are commercialized as racemic agrochemicals and released as equimolar mixtures of enantiomers into the environment. Because of the difference in fungicidal activity and toxicity in enantiomers, stereoselectivity of TF and TN should be an important consideration in ecological risk assessment (Li et al., 2014). Stereoselective behaviors of TF and TN have been reported in soil microorganisms and rainbow trout (Garrison et al., 2011; Kenneke et al., 2010; Li et al., 2011). In our previous study (Wang et al., 2014), we reported the stereoselective metabolism, distribution and bioaccumulation of TF and $\mathrm{TN}$ in lizards under racemic form of TF exposure. These results gave us a preliminary understanding of stereoselective behaviors of TF and TN. However, the chiral conversion between enantiomers and the environmental fate of chiral monomer are still unknown. To fill this gap, we separated the chiral monomers of TF ( $R-(-)$-TF and $S-(+)-\mathrm{TF})$ and used them in the lizard exposure in this paper. Mathematical models for the concentration-time curves were developed and absorption, distribution and elimination rates of $R-(-)-\mathrm{TF}$ and $S-(+)-\mathrm{TF}$ were calculated. The results played an important role in the environmental fate of $\mathrm{TF}$, and gave a suggestion on the use of TF and TN.

\section{Materials and methods}

\subsection{Chemicals and reagents}

Racemic TF (99.55\%) and TN (99.1\%), analytical standards of TN-A (racemate of $R S$ - and $S R$-enantiomer, $99.9 \%$ purity) and TN-B (racemate of $R R$ - and $S S$-enantiomer, $99.9 \%$ purity) were kindly provided by College of Science, China Agricultural University (Beijing, China). The two TF enantiomers were prepared by normal chiral PAK IC Column [cellulose tris-(3, 5-dichlorophenyl-carbamate)] $(4.6 \mathrm{~cm}$ i.d. $25 \mathrm{~cm}$ long) on an Agilent 1260 high performance liquid chromatograph (HPLC) system under the UV detection wave length $225 \mathrm{~nm}$. The mobile phase fraction corresponding to the pure enantiomer was manually collected by observing its UV signal. The purity of separated TF enantiomers were checked with HPLC-MS/MS using the method below (see HPLC-MS/MS conditions). The purity of $R-(-)-\mathrm{TF}$ and $S$ - $(+)-\mathrm{TF}$ were $>96 \%$. Stock solutions of TF and TN were prepared in methanol
(HPLC grade, Dikma, USA) at $1000 \mathrm{mg} / \mathrm{L}$ and kept in dark at $-20^{\circ} \mathrm{C}$.

\subsection{Culture conditions}

The juvenile Eremias argus were obtained from Abag Banner, Inner Mongolia (China), which has no history of chemical application, and maintained in our laboratory since July 2009. Lizards were kept in $5 \times 1.2 \times 0.4 \mathrm{~m}$ solid bottom chamber aquarium covered with $10 \mathrm{~cm}$ of mollisol and fallen leaves. The temperature and humidity were maintained at $25-30{ }^{\circ} \mathrm{C}$ and $30-60 \%$. Daylight lamps (100 W) were set to a 14:10 h light: dark photoperiod to provide enough light and maintain the desired temperature. Lizards were fed with mealworms (Tenebrio molitor) twice a day. The water was sprayed every other day and the excreta and residues were cleaned twice a week.

Sexually mature lizards (3-3.5 g, 2-3 years old) were used in the experiment. The selected lizards were randomly divided into groups of 10 lizards (sex ratio 1: 1). Each group was housed in $30 \times 30 \times 20 \mathrm{~cm}$ glass cage with water pan. The experimental conditions were the same as the feeding conditions. Each lizard was fed two mealworms (about $1.0 \mathrm{~cm}$ each) per day. Animal welfare and experimental procedures were carried out in accordance with the Guide for the Care and Use of Laboratory Animals (Ministry of Science and Technology of China, 2006). The animal care and use procedures were approved by Research Center for Eco-Environmental Sciences, CAS.

\subsection{Dosing and exposure}

Dosing was prepared according to our previous study (Wang et al., 2014). Rac-TF, $R$-(-)-TF and $S$-(+)-TF were first prepared in the ethanol and then dispersed in corn oil. The volume ratio of ethanol to corn oil was 1: 9. To prevent degradation of the compound, the test solution was stored at $4{ }^{\circ} \mathrm{C}$ in a dark place. Before dosing, the ethanol-corn oil lactescence was warmed to room temperature $\left(25^{\circ} \mathrm{C}\right)$ and continually mixed by magnetic stirring apparatus. Micro-syringes were used for oral injection of $30 \mu \mathrm{L}$ corn oil or corn oil-ethanol lactescence to lizard.

The acute oral toxicity of TF to $E$. argus is low toxicity. The safety concentration of $100 \mathrm{mg} / \mathrm{kg}^{\mathrm{bw}}$ was chosen in this study. Rac-TF, $R$ (-)-TF and $S$-(+)-TF were first dispersed into the corn oil-ethanol lactescence and oral injected to lizard. Blood, brain, heart, lungs, liver, kidney and fat were collected for concentration analysis of enantiomers of TF and TN at 2, 6, 8, 10, 12, 24, 48, 72, 120 and $168 \mathrm{~h}$ after lizards were dosed. Three lizards were euthanized with carbon dioxide randomly for each sampling point at once.

\subsection{HPLC-MS/MS conditions}

HPLC was performed using Thermo ACCELA series (Thermo Electron Corporation, Hopkinson, MA) equipped with an ACCELA Autosampler, ACCELA 600 pump, $20 \mu \mathrm{L}$ injection loop and $2 \mu \mathrm{L}$ flow cell. Enantiomers were separated on a Phenomenex Lux Cellulose-1 column $(250 \times 4.6 \mathrm{~mm}$ id, $5 \mu \mathrm{m}$ particles), packed with CSP of CDMPC and obtained from Guangzhou FLM Scientific Instrument (Guangzhou, China). The mobile phase was a mixture of $73 \%$ methanol and $27 \%$ water at a flow rate of $0.5 \mathrm{~mL} / \mathrm{min}$. Chromatographic separation was conducted at $20^{\circ} \mathrm{C}$ with an injection volume of $10 \mu \mathrm{L}$.

TSQ QUANTUM ACCESS MAX was used for LC-MS/MS analysis (Thermo Electron Corporation, Hopkinson, MA). Quantification was performed in positive-ion mode (ESI + ). The signals were received and processed with Thermo Xcalibur 2.2 SP1.48 software. The main working parameters were optimized as follows: Spray Voltage $3200 \mathrm{~V}$, Vaporizer Temperature $250^{\circ} \mathrm{C}$, Sheath Gas Pressure $30 \mathrm{psi}$, Aux Gas Pressure 10 arbitrary units, Capillary Temperature $350{ }^{\circ} \mathrm{C}$, Capillary Offset 35 V, Q2 Collision Gas Pressure 1.5 mTorr. The multiple reaction monitoring mode was used; the precursor and product ions of TF and $\mathrm{TN}$ with the corresponding de-cluster potentials and collision energies are summarized in Supporting information Table S1. 


\subsection{Extraction procedure}

Whole blood (50-100 $\mu \mathrm{L})$ or homogenized tissue matrix $(0.01-0.03 g)$ was transferred to a $2 \mathrm{~mL}$ polypropylene centrifuge tube and $2 \mathrm{~mL}$ acetonitrile (analytical grade, Beijing Chemical Reagent Co. Ltd, China) was added. The mixture was vortexed for $3 \mathrm{~min}$, exposed to ultrasonic vibration for $20 \mathrm{~min}$, and then centrifuged at 10,000 r/min for $5 \mathrm{~min}$. The supernatants were transferred to a separatory funnel.

Samples were re-extracted in the same manner and supernatants were pooled. Five $\mathrm{mL}$ n-hexane (HPLC grade, Dikma, USA) was added for liquid-liquid partitioning to extract most of the lipids. The upper layer of n-hexane was discarded and the acetonitrile layer was collected and evaporated to near dryness using a vacuum rotary evaporator at $30{ }^{\circ} \mathrm{C}$ under a gentle stream of nitrogen. The residue was redissolved in $1 \mathrm{~mL}$ of methanol and filtered through a $0.22-\mathrm{mm}$ filter into a sample vial for HPLC-MS / MS analysis.

\subsection{Data analysis}

The enantiomer fraction (EF) was used to measure the enantioselectivity of TF enantiomers in lizard (Eq. (1)).

$\mathrm{EF}=\frac{\text { concentration of } \mathrm{R}-\mathrm{TF}}{\text { concentration of total } \mathrm{TF}}$

The EF values defined range from 0 to 1 , with $\mathrm{EF}=0.5$ representing the racemic mixture.

Two-compartment model of extravascular administration was used for fitting the blood concentration-time curves of $R$-TF and $S$-TF. For the process (including absorption, distribution, and metabolism) of $R$-TF or $S$-TF, the concentration values of the compartments were calculated as follows.

$\frac{d\left[X_{c}\right]}{d t}=k_{a} X_{a}+k_{p c} X_{p}-k_{c m} X_{c}-k_{c p} X_{p}$

$\frac{d X_{a}}{d t}=F X_{0}-k_{a} X_{a}$

$\frac{d X_{p}}{d t}=k_{c p} X_{c}-k_{p c} X_{p}$

Eqs. (2)-(4) are first order linear non-homogeneous differential equations. For two-compartment model of extravascular administration, the concentration-time equation of central compartment is expressed as

$C=N e^{-k_{a} t}+L e^{-\alpha t}+M e^{-\beta t}$

The kinetic parameters are

$k_{p c}=\frac{L \beta\left(k_{a}-\alpha\right)+M \alpha\left(k_{a}-\beta\right)}{L\left(k_{a}-\alpha\right)+M\left(k_{a}-\beta\right)}$

$k_{c m}=\frac{\alpha \beta}{k_{p c}}$

$k_{c p}=\alpha+\beta-k_{p c}-k_{c e}$

Wherein $N, L$ and $M$ are constants. $C$ represents the concentrations of $R$ $\mathrm{TF}$ or $S$-TF in lizard blood. $K_{a}$ is the absorption rate into blood, $K_{c p}$ is the translation rate from blood to tissue, $K_{p c}$ is the translation rate from tissue to blood, $K_{c m}$ is the metabolism rate in blood. SPSS 16.0 was used for a nonlinear dynamic fitting.

\section{Results and discussion}

\subsection{Method validation}

As shown in Fig. 1, the enantiomers of $\mathrm{TF}$ and $\mathrm{TN}$ got a good separated using the optimized method. The elution order has been described in our previous study (Wang et al., 2014). Enantiomer peaks were shown in Fig. 1. (1) $S R$-(-)-TN (SR-TN), (2) $R S$-(+)-TN (RS-TN), (3) $S S$-(-)-TN (SS-TN), (4) $R R$-(+)-TN (RR-TN4), (5) $R$-(-)-TF ( $R$-TF), (6) $S$ - $(+)$-TF $(S$-TF). $R S$-TN and $S R$-TN were a pair enantiomers of TN-A, which is about $80 \%$ of the total TN. $S S$-TN and $R R$-TN were a pair enantiomers of TN-B, which is about $20 \%$ of the total TN. Linear calibration curves were evaluated over the concentration range of $0.005-5 \mathrm{mg} / \mathrm{L} \quad$ for $\quad R$-TF $\quad(y=733925 x-3659, \quad r=0.9991), \quad S$-TF $(y=755143 x-4197, \quad r=0.9992) \quad$ and $r a c-T F \quad(y=744534 x-7857$, $r=0.9999)$. Linear calibration curves were evaluated over the concentration range of $0.001-2 \mathrm{mg} / \mathrm{L}$ for $S R$-TN $(y=1000000 x-1087$, $r=0.9999), \quad R S$-TN $\quad(y=1000000 x+168.98, \quad r=0.9999), \quad R R$-TN $(y=839672 x-239, r=0.9997)$, SS-TN $(y=826348 x-568, r=0.9999)$. Recovery evaluation was identified at three fortified concentration levels with six replicates. The concentration levels were $0.01,0.1$, and $1 \mathrm{mg} / \mathrm{L}$ (mg/kg in tissue) for rac-TF, $R$-TF and $S$-TF; 0.008, 0.08, and $0.8 \mathrm{mg} / \mathrm{L}(\mathrm{mg} / \mathrm{kg}$ in tissue) for $R S$-TN and $S R-\mathrm{TN}, 0.002,0.02$, and $0.2 \mathrm{mg} / \mathrm{L}(\mathrm{mg} / \mathrm{kg}$ in tissue) for $S S$-TN and $R R$-TN. Recoveries of each enantiomer ranged from $85 \%$ to $107 \%$. The precision of the assay for all enantiomers ranged from $2.5 \%$ to $12.8 \%$ (RSD). LODs for rac-TF, $R$-TF and $S$-TF were $0.003 \mathrm{mg} / \mathrm{kg}$ in lizard tissues and $0.002 \mathrm{mg} / \mathrm{L}$ in lizard blood. LODs for $R S$-TN and $S R$-TN were $0.002 \mathrm{mg} / \mathrm{kg}$ in lizard tissues and $0.002 \mathrm{mg} / \mathrm{L}$ in lizard blood. The LODs for SS-TN and RR-TN were $0.0007 \mathrm{mg} / \mathrm{kg}$ in lizard tissues and $0.0006 \mathrm{mg} / \mathrm{L}$ in lizard blood. The minimum fortified concentration levels in lizard tissues and blood were used as LOQs for all enantiomers.

\subsection{Time-concentration curve in lizard tissues}

After oral administration of $R$-TF, $S$-TF and rac-TF, time-concentration curve of TF enantiomers in lizard tissues were shown in Fig. 2. The times to reach maximum concentration in different tissues in $R$-TF exposure group were little different from that in $S$-TF exposure group. However, there were some similarities in the concentration-time curves of the two exposed groups in different tissues. Both in $R$-TF and $S$-TF groups, the concentrations first reached the maximum values immediately at $2 \mathrm{~h}$ in blood after oral injection. The times to reach the maximum concentration in other tissues were later than that in the blood. This provides the basis for our understanding of blood as a central compartment in the following model calculations. In all tissues, the highest maximum concentration was detected in brain indicating that the blood encephalopathy barrier does not affect the absorption of the TF enantiomer in the brain. It has been reported that TF can induce rodent neurobehavioral effects (Perkins et al., 1992). Acute exposure to TF affects catecholamines in the central nervous system and induces transient syndromes involving hyperactivity and stereotyping in rats (Moser and Macphail, 1989). These results suggest that the brain is one of the target organs of TF, which consistent with the results of our study. Possible reasons may be that TF can act as an inhibitor of dopamine transporter, by binding dopamine transporter and increasing the level of synaptic DA as an indirect dopamine (DA) agonist (Gagnaire and Micillino, 2006; Reeves et al., 2004). The maximum concentration value times in liver and kidney were 4-6 h after oral injection in two exposure groups. After reaching the highest values, the TF enantiomers concentrations declined rapidly in liver and kidney. The reason might be that liver and kidney were considered to be primary organs of metabolism and exclusion. Furthermore, high concentration values of $R$-TF and $S$-TF were detected in the liver between 2 and $8 \mathrm{~h}$. In the process of transformation of TF enantiomers in the liver might also produce damage to the liver. Previous study showed that triazole pesticides might reduce cholesterol concentration, which is thought to be an early indication of liver dysfunction. And may have a causal relationship with subsequent pathological changes as well as final cell death, increased cell proliferation and tumor formation (Allen et al., 2006). These results indicated that liver was also the target organ of TF. The TF enantiomers concentrations in lung were lowest among the tissues and the maximum value times in lung were $6-10 \mathrm{~h}$ after oral 

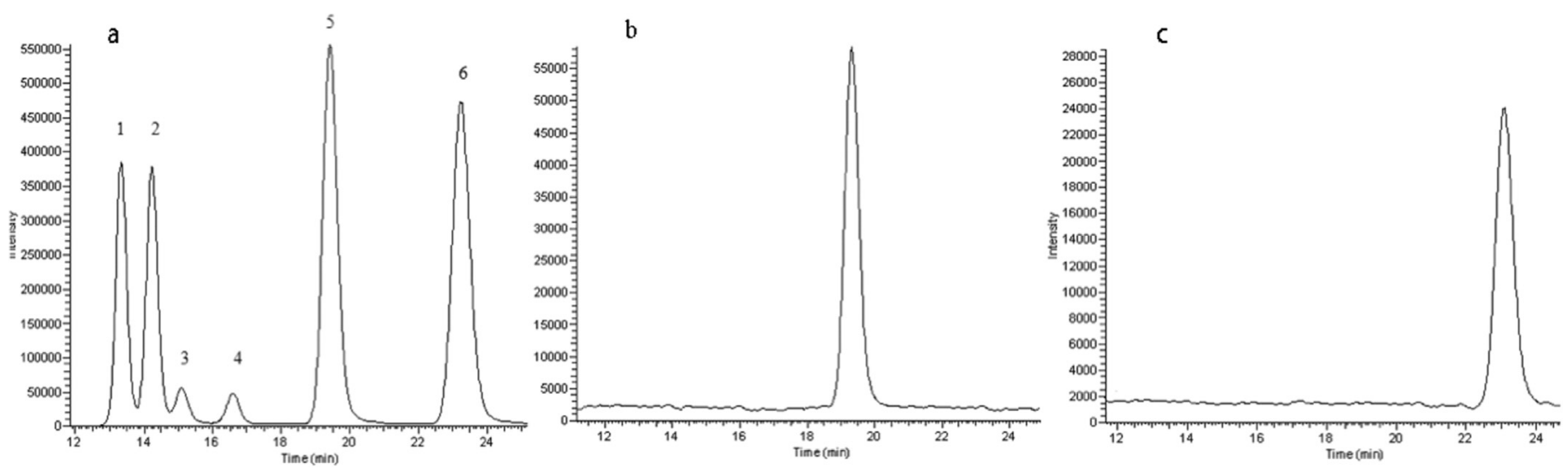

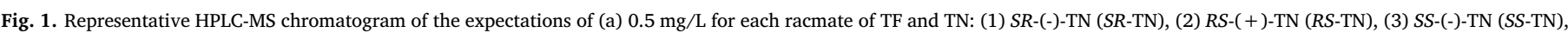
(4) $R R$ - $(+)-\mathrm{TN}(R R$-TN4), (5) $R$-(-)-TF ( $R$-TF), (6) $S$ - $(+)$-TF ( $S$-TF), (b) separated enantiopure $R$-(-)-TF, (c) separated enantiopure $S$ - $(+)-\mathrm{TF}$.

injection. Blood flow might also be the reason caused the difference of concentrations between tissues. The trends of TF enantiomers concentrations in fat were different from that in other tissues. The TF enantiomers concentrations in fat first increased slowly and reach the maximum values at about $24 \mathrm{~h}$. After $24 \mathrm{~h}$, the TF enantiomers concentrations declined very slowly and maintained stable after about $72 \mathrm{~h}$. Compare to other tissues, the maximum value times in fat were longer and the ending concentrations were much higher. This phenomenon illustrated that fat was the main organ of accumulation.

In blood, brain and liver, the maximum concentration values in $R$ TF exposure group were all higher than that in $S$-TF exposure group ( $t$ test, $p<0.05$ ). But in other tissues, the highest concentrations did not show significant difference between $R$-TF and $S$-TF exposure groups $(t-$ test, $p>0.05$ ). In rac-TF exposure group, the $R$-TF concentrations in all tissues were higher than $S$-TF concentrations at the same sampling time ( $t$-test, $p<0.05$ ). The average EF values in different tissues and blood were calculated in rac-TF exposure group. EF values in all tissues and blood were higher than 0.5 that verified the stereoselectivity of TF (data not shown). Simple $t$-tests were used to compare the difference among the EF values of TF1 in tissues and blood. The highest EF value were
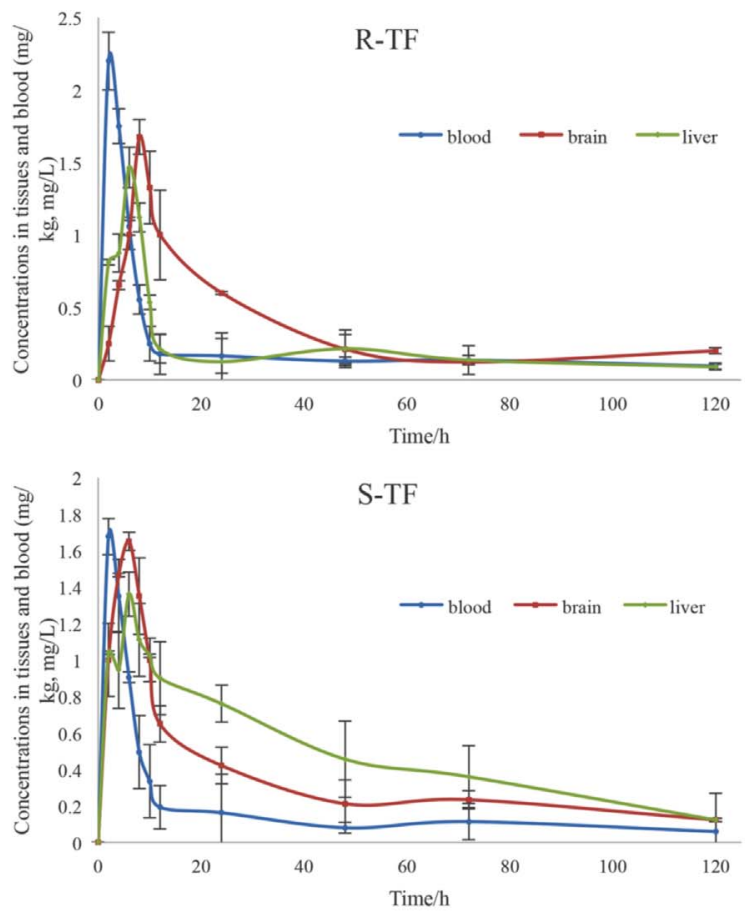

detected in liver $(\mathrm{EF}=0.64)$ that showed liver was the main stereoselective metabolism organ. The lowest EF values were detected in kidney and fat $(\mathrm{EF}=0.55)$. Considering that stereroselective exclusion and accumulation predominate in kidney and fat, respectively, the lowest EF values in kidney and fat indicated that the difference between TF enantiomers became smaller in the exclusion and accumulation process.

\subsection{Kinetic considerations}

While TF enantiomers were oral injected into lizard, it would first uptake into absorption compartment (intestines and stomach) and then absorbed immediately in blood (central compartment). Through the blood circulation, TF enantiomers were distributed into each tissue (peripheral compartment). This process was described in Fig. 3. The blood concentration-time curves of $R$-TF and $S$-TF (as shown in Fig. 2) were consistent with two-compartment model of extravascular administration. The fitting results were shown in Table 1. Normally in the Eq. (5), $K_{a}$ is used to reflect the absorption rate. $K_{a}$ of $S$-TF (0.627) was higher than $K_{a}$ of $R$-TF (0.450), which meant $S$-TF was absorbed faster
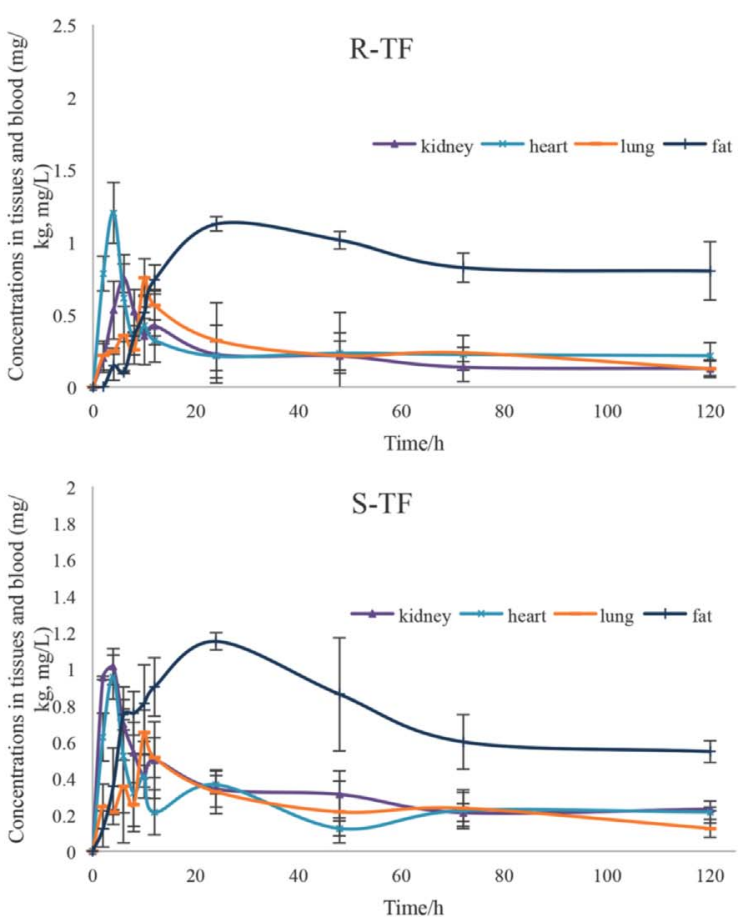

Fig. 2. Time-concentration curve in lizard tissues in $R$-TF and $S$-TF exposure group, respectively. 


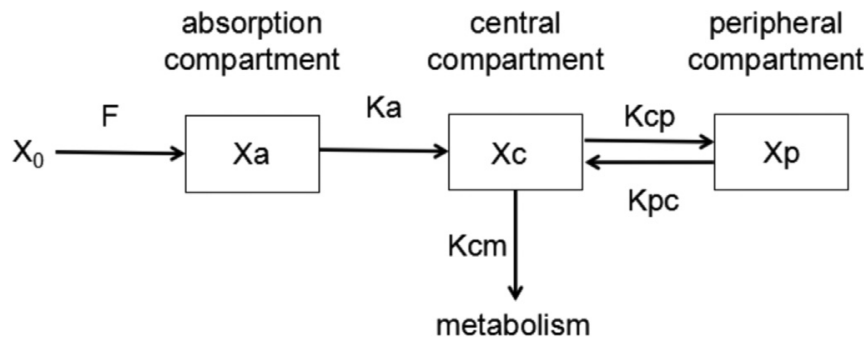

Fig. 3. The absorption, distribution and elimination process of TF in lizard.

than $R$-TF. Parameter $\alpha$ is used to reflect the distribution rate. The $\alpha$ of $R$-TF (0.378) was higher than the $\alpha$ of $S$-TF (0.264), which indicated $R$ TF was easily distributed in the tissues. Parameter $\beta$ is used to reflect the elimination rate. The $\beta$ of $S$-TF (0.687) was higher than the $\beta$ of $R$-TF (0.427), which meant that $S$-TF was eliminated faster than $R$-TF. In $R$ $\mathrm{TF}$ and $S$-TF exposure groups, the $K_{p c}$ values were much higher than $K_{c p}$ values. The reason caused this result might be the concentrations declined rapidly in blood and $\mathrm{TF}$ enantiomers transported easily back into blood from the tissues. The $K_{c m}$ value of $R$-TF (0.475) was higher than that of $S$-TF (0.315), which meant $R$-TF was more easily converted into its metabolites. The differences in $R$-TF and $S$-TF concentrations in lizards were a combined effect of absorption, metabolism, exclusion, and distribution.

\subsection{Metabolic transformation of TF to TN}

The metabolic transformation of TF to TN has been shown in Fig. 4. TF has only one chiral center and consists of an equimolecular mixture of the two enantiomers, $R$-TF and $S$-TF. During the metabolic transformation, the carbonyl group connected on the chiral center is converted to a hydroxyl group, resulting in a new chiral center. So TN has two chiral centers and consists of four stereoisomers including two pairs of diastereomers, TN-A and TN-B. $R S$-TN and $S R$-TN are a pair of enantiomers of TN-A, $R R$-TN and $S S$-TN are a pair of enantiomers of TN-B. However, from the chemical structure analysis, $R S$-TN and $R R$-TN are the metabolite of $R$-TF, and $S R$-TN and $S S$-TN are the metabolite of $S$-TF. This result means that one metabolite of $R$-TF and one metabolite of $S$-TF constitute a pair of chiral enantiomers. From the results of exposure test of $R$-TF and $S$-TF, we detected the theoretically two TN metabolites of $R$-TF and $S$-TF, respectively. Furthermore, no $S R$-TN and $S S$-TN were detected in $R$-TF exposure group, and no $R S$-TN and $R R$-TN were detected in $S$-TF exposure group. This results indicated that chiral conversion between the theoretically two TN metabolites in each TF exposure group possible be existed, but the chiral conversion from $R R$ TN, $R S$-TN to $S S$-TN, $S R$-TN were not existed, vice versa.

\subsection{Discussion}

There is no eco-toxicology data on TF and its enantiomers. Previous study showed that $R$-TF is more toxic than the rac-TF (about 2-fold) to blackfly larvae (Kellock K et al., 2009). In soil, TN shows a much longer persistence (soil half-life $>240$ days) than TF (half-life of 6-30 days)
(Bromilow et al., 1999; Singh, 2005). The risk of converted TN from TF shouldn't be ignored while evaluating the risk of TF. The $S R$-TN (produced by $S$-TF) has the highest fungicidal activity (up to 1000 -fold greater than the other three enantiomers) (Burden et al., 1987). But $S R$ TN has the greatest inhibition of sterol synthesis in yeast at the same time (Yoshida and Aoyama, 1990). Fungicidal activity and toxicity were two opposite factors in the risk assessment. The fungicidal activity, eco-toxicity and the accumulation should be comprehensive considered in the risk assessment. During the 35 days of continuous exposure of $\mathrm{rac}$-TF in lizard, concentrations of metabolic product $R R$-TN were much higher than the other three enantiomers (Wang et al., 2014). The $R R$-TN metabolized from $R$-TF accounted for about $70 \%$ of TN overall ratio. The easy accumulation of $R R$-TN added the risk of the use of $R$-TF. In the four enantiomers of TN, $S R$-TN produced by $S$-TF may have the strongest biological toxicity; $R R$-TN produced by $R$-TF is most likely to occur bioaccumulation effect in lizard. Identifying toxicological effects and dose-response relationship of $S R$-TN and $R R$ TN will help us to fully assess the risk of TF enantiomers use in the future.

\section{Conclusions}

According to the findings of this study, after single oral administration of $R$-TF and $S$-TF, the maximum value times in different tissues were found to be different. TF enantiomers could cross the blood-brain barrier easily and brain is the main target organ. Blood flow might be the reason caused the difference of concentrations between tissues. The residue of TF enantiomers in fat were much higher than other tissues, fat was the main organ of accumulation.

In rac-TF exposure groups, the EF values of TF1 in all tissues and blood were significant different from its nature ratio $0.5(p<0.05)$. These results indicated stereoslectivity of TF occurred in the process of absorption, distribution, metabolism and exclusion in lizard. Simple $t$ tests were used to compare the difference among the EF values of TF1 in tissues and blood. The highest EF values were detected in liver and the lowest EF values were detected in kidney and fat. The difference between liver and kidney (or fat) were significant $(p<0.05)$. Stereoselective differences were significant in absorption and metabolism, but the differences became smaller in exclusion and accumulation.

From the results of mathematical models, $S$-TF was absorbed and eliminated faster than $R$-TF, and $R$-TF was easily distributed in the tissues and more easily converted into its metabolites. The risk of converted TN from TF shouldn't be ignored while evaluating the risk of TF. The $S R$-TN (produced by $S$-TF) has the highest fungicidal activity and strongest biological toxicity. High fungicidal activity could reduce the usage amount of pesticide, but the strong toxicity may cause toxic reactions in a small residue concentration. The $R R$-TN (produced by $R$ TF) has been tested most likely to occur bioaccumulation effect in lizard. This may result in accumulation effect and increase the risk of pesticide use. The fungicidal activity, eco-toxicity and the accumulation should be comprehensive considered in the risk assessment. Identifying toxicological effects and dose-response relationship of $S R$-TN and $R R$ TN will help us to fully assess the risk of TF enantiomers use in the

Table 1

The kinetic parameters of TF enantiomers after oral administration in lizard.

\begin{tabular}{lll}
\hline & $R$-TF group & $S$-TF group \\
\hline Function & $\mathrm{C}=10.429 \mathrm{e}^{-0.450 \mathrm{t}}+12.028 \mathrm{e}^{-0.378 \mathrm{t}}-22.458 \mathrm{e}^{-0.536 \mathrm{t}}$ & $\mathrm{C}=5.212 \mathrm{e}^{-0.627 \mathrm{t}}+4.472 \mathrm{e}^{-0.264 \mathrm{t}}-9.684 \mathrm{e}^{-0.687 \mathrm{t}}$ \\
$\mathrm{R}^{2}$ & 0.998 & 0.996 \\
$K_{a}\left(\mathrm{mg} / \mathrm{L} \mathrm{h}^{-1}\right)$ & 0.450 & 0.627 \\
$\alpha\left(\mathrm{mg} / \mathrm{L} \mathrm{h}^{-1}\right)$ & 0.378 & 0.264 \\
$\beta\left(\mathrm{mg} / \mathrm{L} \mathrm{h}{ }^{-1}\right)$ & 0.427 & 0.687 \\
$K_{c p}\left(\mathrm{mg} / \mathrm{L} \mathrm{h}^{-1}\right)$ & 0.012 & 0.060 \\
$K_{p c}\left(\mathrm{mg} / \mathrm{L} \mathrm{h}^{-1}\right)$ & 0.427 & 0.576 \\
$K_{c m}\left(\mathrm{mg} / \mathrm{L} \mathrm{h} \mathrm{h}^{-1}\right)$ & 0.475 & 0.315 \\
\hline
\end{tabular}




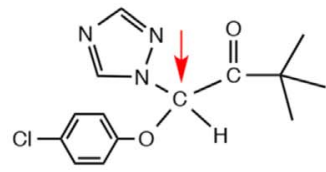

Triadimefon(TF)

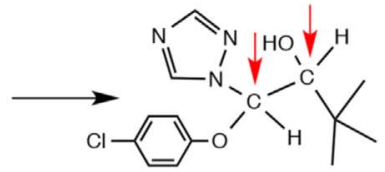

Triadimenol(TN)

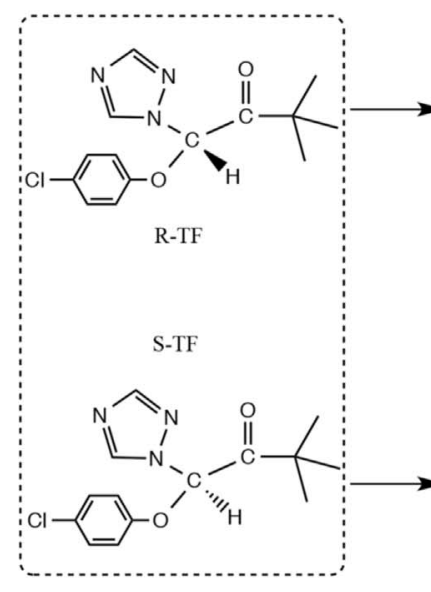

TF

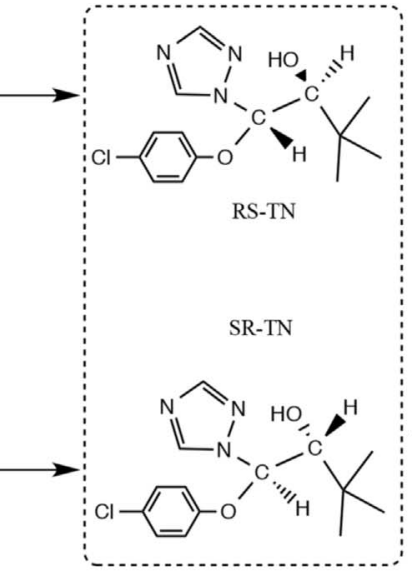

TN-A

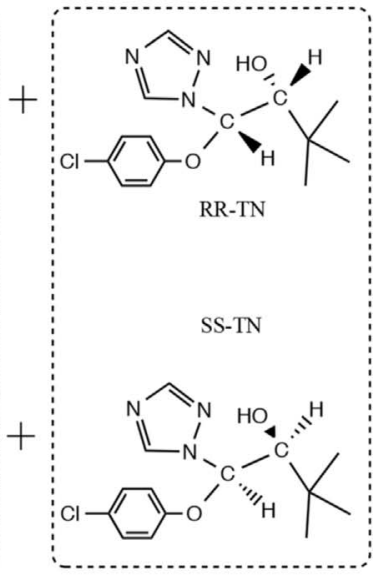

TN-B

Fig. 4. The metabolic transformation of TF to TN.

future.

\section{Acknowledgements}

The present study was supported by the National Natural Science Foundation of China (Contract Grant nos. 41301569, 21277163, and 21477152).

\section{Appendix A. Supporting information}

Supplementary data associated with this article can be found in the online version at http://dx.doi.org/10.1016/j.ecoenv.2017.05.024.

\section{References}

Allen, J.W., Wolf, D.C., George, M.H., et al., 2006. Toxicity profiles in mice treated with hepatotumorigenic and non-hepatotumorigenic triazole conazole fungicides: propiconazole, triadimefon, and myclobutanil. Toxicol. Pathol. 34, 853-862.

Brandelavridsen, N., Christensendalsgaard, J., Korsgaard, B., 2008. Effects of prochloraz and ethinylestradiol on sexual development in Rana temporaria. J. Exp. Zool. Part A Ecol. Genet. Physiol. 309, 389-398.

Bromilow, R.H., Evans, A.A., Nicholls, P.H., 1999. Factors affecting degradation rates of five triazole fungicides in two soil types: 1. Lab. Incubations Pestic. Sci. 55, $1135-1142$.

Buono, S., Cristiano, L., D'Angelo, B., et al., 2007. PPARalpha mediates the effects of the pesticide methyl thiophanate on liver of the lizard Podarcis sicula. Comp. Biochem. Physiol. Part C Toxicol. Pharmacol. 145, 306-314.

Burden, R.S., Carter, G.A., Clark, T., et al., 1987. Comparative activity of the enantiomers of triadimenol and paclobutrazol as inhibitors of fungal growth and plant sterol and gibberellin biosynthesis. Pestic. Sci. 21, 253-267.

Buser, H.R., Mueller, M.D., Rappe, C., 1992. Enantioselective determination of chlordane components using chiral high-resolution gas chromatography-mass spectrometry with application to environmental samples. Environ. Sci. Technol. 26, 325-330.

Cai, X., Liu, W., Sheng, G., 2008. Enantioselective degradation and ecotoxicity of the chiral herbicide diclofop in three freshwater alga cultures. J. Agric. Food Chem. 56, 2139-2146.

Crowell, S.R., Henderson, W.M., Kenneke, J.F., et al., 2011. Development and application of a physiologically based pharmacokinetic model for triadimefon and its metabolite triadimenol in rats and humans. Toxicol. Lett. 205, 154-162.

Dong, F., Li, J., Chankvetadze, B., et al., 2013. Chiral triazole fungicide difenoconazole: absolute stereochemistry, stereoselective bioactivity, aquatic toxicity, and environmental behavior in vegetables and soil. Environ. Sci. Technol. 47, 3386-3394.

Gagnaire, F., Micillino, J.C., 2006. Effects of triadimefon on extracellular dopamine, DOPAC, HVA and 5-HIAA in adult rat striatum. Toxicology 217, 91-104.

Garrison, A.W., Avants, J.K., Jones, W.J., 2011. Microbial transformation of triadimefon to triadimenol in soils: selective production rates of triadimenol stereoisomers affect exposure and risk. Environ. Sci. Technol. 45, 2186-2193.

Gervais, J.A., 2011. Ecotoxicology of Amphibians and Reptiles. Second edition by Donald W. Sparling; Greg Linder; Christine A. Bishop; Sherry K. Krest. Journal of Wildlife Management, vol. 75, pp. 749-750.

Gibbon, J.W., Scott, D.E., Ryan, T.J., et al., 2000. The Global Decline of Reptiles, Déjà Vu Amphibians. BioScience 50, 653-666.

Holem, R.R., Hopkins, W.A., Talent, L.G., 2008. Effects of repeated exposure to malathion on growth, food consumption, and locomotor performance of the western fence lizard (Sceloporus occidentalis). Environ. Pollut. 152, 92-98.

Kellock K, O.J., Garrison A.W., Avants, J.K., 2009. Toxicity of triadimefon racemate and enantiomers to black fly larvae. In: Proceedings of the SETAC North America 30th Annual Meeting. New Orleans, LA, pp. 296-297.

Kenneke, J.F., Ekman, D.R., Mazur, C.S., et al., 2010. Integration of metabolomics and in vitro metabolism assays for investigating the stereoselective transformation of triadimefon in rainbow trout. Chirality 22, 183-192.

Kenneke, J.F., Mazur, C.S., Kellock, K.A., et al., 2009. Mechanistic approach to understanding the toxicity of the azole fungicide triadimefon to a nontarget aquatic insect and implications for exposure assessment. Environ. Sci. Technol. 43, 5507-5513.

Li, Y., Dong, F., Liu, X., et al., 2014. Chiral fungicide triadimefon and triadimenol: stereoselective transformation in greenhouse crops and soil, and toxicity to Daphnia magna. J. Hazard. Mater. 265, 115-123.

Li, Z., Zhang, Y., Li, Q., et al., 2011. Enantioselective degradation, abiotic racemization, and chiral transformation of triadimefon in soils. Environ. Sci. Technol. 45, 2797-2803.

Liang, H., Li, L., Qiu, J., et al., 2013. Stereoselective transformation of triadimefon to metabolite triadimenol in wheat and soil under field conditions. J. Hazard. Mater. 260, 929-936.

Lin, L., Yutao, Z., Jinzhong, Z., 2008. Research progress on the residue analysis of triazole pesticides. J. Anhui Agric. Sci. 36 (22), 9704-9707.

Menegola, E., Broccia, R.F., Prati, M., et al., 2000. In vitro teratogenic potential of two antifungal triazoles: triadimefon and triadimenol. Vitr. Cell. Dev. Biol. Anim. 36, 88-95.

Merwe, J.P.V.D., Hodge, M., Olszowy, H.A., et al., 2009. Using blood samples to estimate persistent organic pollutants and metals in green sea turtles (Chelonia mydas). Mar. Pollut. Bull. 60, 579-588.

Moser, V.C., Macphail, R.C., 1989. Neurobehavioral effects of triadimefon, a triazole fungicide, in male and female rats. Neurotoxicol. Teratol. 11, 285-293.

Moss, S., Keller, J.M., Richards, S., et al., 2009. Concentrations of persistent organic pollutants in plasma from two species of turtle from the Tennessee River Gorge. Chemosphere 76, 194-204.

Perkins, A.N., Eckerman, D.A., Macphail, R.C., 1992. Discriminative stimulus properties of triadimefon: comparison with methylphenidate. Pharmacol. Biochem. Behav. 40, 757-761.

Qi, Y., Liu, D., Luo, M., Jing, X., Wang, P., Zhou, Z., 2016. Enantioselective degradation and chiral stability of the herbicide fluazifop-butyl in soil and water. Chemosphere $146,315-322$.

Randhawa, M.A., Anjum, M.N., Butt, M.S., Yasin, M., Imran, M., 2014. Minimization of imidacloprid residues in cucumber and bell pepper through washing with citric acid and acetic acid solutions and their dietary intake assessment. Int. J. Food Prop. 17, 978-986. 
Reeves, R., Thiruchelvam, M., Richfield, E.K., et al., 2004. The effect of developmental exposure to the fungicide triadimefon on behavioral sensitization to triadimefon during adulthood. Toxicol. Appl. Pharmacol. 200, 54-63.

Roberts, T.R., Hutson, D.H., 1999. Metabolic pathways of agrochemicals. Part 2: Insecticides and Fungicides. R. Soc. Chem.

Simoniello, P., Filosa, S., Riggio, M., et al., 2010. Responses to cadmium intoxication in the liver of the wall lizard Podarcis sicula. Comp. Biochem. Physiol. Part C Toxicol. Pharmacol. 151, 194-203.

Singh, N., 2005. Factors affecting triadimefon degradation in soils. J. Agric. Food Chem. $53,70-75$.

U.S. Environmental Protection Agency (U.S. EPA), 2006. Reregistration Eligibility Decision for Triadimefon and Tolerence Reassessment for Triadimenol, EPA 738-R06-003. U.S. Environmental Protection Agency, Washington,DC.

Ulrich, E.M., Morrison, C.N., Goldsmith, M.R., et al., 2012. Chiral pesticides: identification, description, and environmental implications. Rev. Environ. Contam. Toxicol. 217, 1-74.

Vinggaard, A.M., Hnida, C., Breinholt, V., et al., 2000. Screening of selected pesticides for inhibition of CYP19 aromatase activity in vitro. Toxicol. Vitr. 14, 227-234.

Wang, Y., Yu, D., Xu, P., et al., 2014. Stereoselective metabolism, distribution, and bioaccumulation brof triadimefon and triadimenol in lizards. Ecotoxicol. Environ. Saf. 107C, 276-283.
Wagner, N., Mingo, V., Schulte, U., et al., 2015. Risk evaluation of pesticide use to protected European reptile species. Biol. Conserv. 191, 667-673.

Weir, S.M., Suski, J.G., Salice, C.J., 2010. Ecological risk of anthropogenic pollutants to reptiles: evaluating assumptions of sensitivity and exposure. Environ. Pollut. 158, 3596-3606.

Wong, C.S., 2006. Environmental fate processes and biochemical transformations of chiral emerging organic pollutants. Anal. Bioanal. Chem. 386, 544-558.

Ye, J., Zhao, M., Liu, J., et al., 2010. Enantioselectivity in environmental risk assessment of modern chiral pesticides. Environ. Pollut. 158, 2371-2383.

Yoshida, Y., Aoyama, Y., 1990. Stereoselective interaction of an azole antifungal agent with its target, lanosterol 14 $\alpha$-demethylase (cytochrome P-45014DM): a model study with stereoisomers of triadimenol and purified cytochrome P-45014DM from yeast. Chirality 2, 10-15.

Zadra, C., Marucchini, C., Zazzerini, A., 2002. Behavior of Metalaxyl and its pure REnantiomer in sunflower plants (Helianthus annus). J. Agric. Food Chem. 50, 5373-5377.

Zhang, Q., Gao, B., Tian, M., Shi, H., Hua, X., Wang, M., 2016. Enantioseparation and determination of triticonazole enantiomers in fruits, vegetables, and soil using efficient extraction and clean-up methods. J. Chromatogr. B 1009, 130-137.

Zhang, W.H., Yang, C.L., Wang, M.H., et al., 2003. Application of catalytic asymmetric reactions to the synthesis of chiral pesticides. Chin. J. Org. Chem. 23, 741-749. 